\title{
VARIANTS OF THE MAXIMAL DOUBLE HILBERT TRANSFORM
}

BY

ELENA PRESTINI

\begin{abstract}
We prove the boundedness on $L_{p}\left(T^{2}\right), 1<p<\infty$, of two variants of the double Hilbert transform and maximal double Hilbert transform. They have an application to a problem of almost everywhere convergence of double Fourier series.
\end{abstract}

Introduction. In this paper we study two variants of the double Hilbert transform

$$
D f(x, y)=\iint_{\left|y^{\prime}\right| \leq \pi,\left|x^{\prime}\right| \leq \pi} 1 / x^{\prime} y^{\prime} f\left(x-x^{\prime}, y-y^{\prime}\right) d x^{\prime} d y^{\prime}
$$

and maximal double Hilbert transform

$$
\tilde{D} f(x, y)=\operatorname{Sup}_{\varepsilon, \delta>0}\left|\iint_{\delta<\left|y^{\prime}\right| \leq \pi, \varepsilon<\left|x^{\prime}\right| \leq \pi} 1 / x^{\prime} y^{\prime} f\left(x-x^{\prime}, y-y^{\prime}\right) d x^{\prime} d y^{\prime}\right|
$$

which are, roughly speaking, of the following kind. First we consider

$$
H_{1} f(x, y)=\iint_{\left(x^{\prime}, y^{\prime}\right) \in A} 1 / x^{\prime} y^{\prime} f\left(x-x^{\prime}, y-y^{\prime}\right) d x^{\prime} d y^{\prime}
$$

and

$$
\tilde{H}_{1} f(x, y)=\operatorname{Sup}_{\varepsilon, \delta>0}\left|\iint_{\substack{\left(x^{\prime}, y^{\prime}\right) \in A \\\left|x^{\prime}\right|>\varepsilon,\left|y^{\prime}\right|>\delta}} 1 / x^{\prime} y^{\prime} f\left(x-x^{\prime}, y-y^{\prime}\right) d x^{\prime} d y^{\prime}\right|,
$$

where $A \subset\left\{\left(x^{\prime}, y^{\prime}\right):\left|x^{\prime}\right| \leq \pi,\left|y^{\prime}\right| \leq \pi\right\}=T^{2}$ is a fixed region symmetrical with respect to the axes $x^{\prime}$ and $y^{\prime}$ but, except for this natural requirement, quite general. (The cut-off of the kernel $1 / x^{\prime} y^{\prime}$, given by $\chi_{A}\left(x^{\prime}, y^{\prime}\right)$, is actually smoothly done. See $\S 2$ for the exact definition.) Secondly, we consider

$$
H_{2} f(x, y)=\iint_{\left(x^{\prime}, y^{\prime}\right) \in A_{x}} 1 / x^{\prime} y^{\prime} f\left(x-x^{\prime}, y-y^{\prime}\right) d x^{\prime} d y^{\prime}
$$

and

$$
\tilde{H}_{2} f(x, y)=\operatorname{Sup}_{\delta>0}\left|\iint_{\substack{\left(x^{\prime}, y^{\prime}\right) \in A_{x} \\\left|y^{\prime}\right|>\delta}} 1 / x^{\prime} y^{\prime} f\left(x-x^{\prime}, y-y^{\prime}\right) d x^{\prime} d y^{\prime}\right|,
$$

where, for every $x$, the domain of integration $A_{x}$ is symmetrical with respect to the axes and otherwise is quite general.

Received by the editors November 28, 1983 and, in revised form, June 26, 1984 .

1980 Mathernatics Subject Classification. Primary 42A40; Secondary 42A92.

(C)1985 American Mathematical Society $0002-9947 / 85 \$ 1.00+\$ .25$ per page 
We shall prove that $H_{1}, \tilde{H}_{1}, H_{2}, \tilde{H}_{2}$ are bounded operators from $L_{p}\left(T^{2}\right)$ to itself, $1<p<\infty$. Moreover, we shall give a pointwise estimate from above of $\tilde{H}_{1}$ and $\tilde{H}_{2}$ similar to the known one concerning $\tilde{D}$ (see [6, p. 218]). Namely, we are going to prove that

$$
\begin{gathered}
\tilde{H}_{1} f(x, y) \leq c\left\{M_{x^{\prime}} M_{y^{\prime}} f(x, y)+M_{x^{\prime}} \tilde{H}_{y^{\prime}} f(x, y)\right. \\
\left.\quad+M_{y^{\prime}} \tilde{H}_{x^{\prime}} f(x, y)+M_{x^{\prime}} M_{y^{\prime}}\left(H_{1} f\right)(x, y)\right\} \\
\tilde{H}_{2} f(x, y) \leq c\left\{M_{y^{\prime}} \tilde{H}_{x^{\prime}} f(x, y)+M_{\bar{y}}\left(H_{2} f(x, \bar{y})\right)(y)\right\}
\end{gathered}
$$

where $M_{x^{\prime}}$ is the Hardy-Littlewood maximal function acting on the $x^{\prime}$ variable, $\tilde{H}$ denotes a variant of the maximal Hilbert transform (see $\S 1$ ). These results apply to a problem of almost everywhere convergence of double Fourier series [5], where it appears that $\tilde{H}_{1}$ and $\tilde{H}_{2}$ play the same central role that the maximal Hilbert transform plays in the proof of a.e. convergence of Fourier series of one variable $[\mathbf{1}$, $\mathbf{2}, \mathbf{4}]$.

Let us observe that $H_{1}$ and $\tilde{H}_{1}$ fall under the scope of Theorems 2 and 4 of [3]. The proof given in [3] of Theorem 4 uses complex interpolation and it is quite technical. Ours involves only elementary estimates; moreover, we are able to control $\tilde{H}_{1}$ from above by proving (1). This is most important for the mentioned application and it is not proved in [3].

The paper is structured as follows. In $\S 1$ we are concerned with the onedimensional case and with the maximal Carleson operator. In $\S \S 2$ and 3, respectively, we study $H_{1}, \tilde{H}_{1}$ and $H_{2}, \tilde{H}_{2}$. In $\S 4$ we consider an even more general operator $\tilde{H}_{3}$ (where the Sup is taken over all regions). We give a counterexample to show that $\tilde{H}_{3}$ is not a bounded operator. This sets a halt to our generalisations of the maximal double Hilbert transform. Finaly, in $\S 5$ we say some more about the application we mentioned.

By $c$ we denote a constant not necessarily the same in all instances.

1. The one-dimensional case. There exists a $C^{\infty}$ function $\phi\left(x^{\prime}\right)$ supported on $\left\{\left|x^{\prime}\right| \leq 2 \pi\right\}$ such that if we write $\phi_{k}\left(x^{\prime}\right)=2^{k} \phi\left(2^{k} x^{\prime}\right)$, then $1 / x^{\prime}=\sum_{k=0}^{\infty} \phi_{k}\left(x^{\prime}\right)$ for $\left|x^{\prime}\right| \leq \pi$. Let $J$ be a fixed subset of the nonnegative integers $N$ and let us consider the operators

$$
H f(x, y)=\int \sum_{k \in J} \phi_{k}\left(x^{\prime}\right) f\left(x-x^{\prime}\right) d x^{\prime}
$$

and

$$
\tilde{H} f(x)=\operatorname{Sup}_{K>0}\left|\int \sum_{\substack{k \in J \\ k \leq K}} \phi_{k}\left(x^{\prime}\right) f\left(x-x^{\prime}\right) d x^{\prime}\right|
$$


$\tilde{H}$ is clearly a variant of the maximal Hilbert transform. We have

LEMMA 1. $H$ and $\tilde{H}$ are bounded operators on $L_{p}(T), 1<p<\infty$, with norm independent of $J$. Moreover, the following inequality holds:

$$
\tilde{H} f(x) \leq c\{M f(x)+M(H f)(x)\} .
$$

REMARK. This is exactly Lemma 3 of [2]. We are going to prove it for the reader's convenience and for an inaccuracy that appears in [2]. Namely, one needs to use a smooth cut-off function like the following $\theta\left(x^{\prime}\right)$ rather than a sharp one.

PROOF. Clearly, $\phi$ has the following properties:

1. $\hat{\phi}(0)=0$,

2. $|\hat{\phi}(\xi)| \leq c_{M} /|\xi|^{M}$ for $|\xi|>1$ and for any integer $M \geq 0$,

3. $|\hat{\phi}(\xi)| \leq c|\xi|$ for $|\xi| \leq 1$.

Since $\hat{\phi}_{k}(\xi)$ is mainly supported on $|\xi| \sim 2^{k}$, we have that

$$
\left|m_{K}(\xi)\right|=\left|\sum_{\substack{k \in J \\ k \leq K}} \hat{\phi}_{k}(\xi)\right| \leq \sum_{k=0}^{\infty}\left|\hat{\phi}_{k}(\xi)\right| \leq c
$$

independently of $J$ and $K$. We have $m_{K}^{\prime}(\xi)=\sum_{k \in J, k<K} \hat{\psi}_{k}(\xi)$, where $\psi\left(x^{\prime}\right)=$ $x^{\prime} \phi\left(x^{\prime}\right)$ and $\psi_{k}\left(x^{\prime}\right)=2^{k} x^{\prime} \phi\left(2^{k} x^{\prime}\right)=\psi\left(2^{k} x^{\prime}\right)$. Now $\psi\left(x^{\prime}\right)$ is $C^{\infty}$, compactly supported and, moreover,

4. $|\hat{\psi}(\xi)| \leq c$ for every $\xi$,

5. $|\hat{\psi}(\xi)| \leq c_{M} /|\xi|^{M}$ for $|\xi|>1$ and for any integer $M \geq 0$.

Therefore, for any dyadic interval $I \subset \mathbf{R}$ we have that

$$
\int_{I}\left|m_{K}^{\prime}(\xi)\right| d \xi \leq \int_{I} \sum_{k=0}^{\infty}\left|\hat{\psi}_{k}(\xi)\right| d \xi \leq c .
$$

By the Marcinkiewicz multiplier theorem, the operator

$$
H_{K} f(x)=\int \sum_{\substack{k \in J \\ k \leq K}} \phi_{k}\left(x^{\prime}\right) f\left(x-x^{\prime}\right) d x^{\prime}
$$

is bounded on $L_{p}$ with norm independent of $K$. By a standard argument there exists $\lim _{K \rightarrow \infty} H_{K} f(x)=H f(x)$ in $L_{p}$ norm and $\|H f\|_{p} \leq c_{p}\|f\|_{p}, 1<p<\infty$.

Now let $\theta\left(x^{\prime}\right)$ be a positive $C^{\infty}$ function supported on $\left\{\left|x^{\prime}\right| \leq 1\right\}$ and such that $\int_{-1}^{1} \theta\left(x^{\prime}\right) d x^{\prime}=1$. To prove equation (3) we are going to show that

$$
\left|\sum_{\substack{k \in J \\ k \leq K}} \phi_{k}\left(x^{\prime}\right)-\theta_{K} * \sum_{k \in J} \phi_{k}\left(x^{\prime}\right)\right| \leq c 2^{-K} /\left(x^{\prime}\right)^{2}+2^{-2 K},
$$

where $\theta_{K}\left(x^{\prime}\right)=2^{K} \theta\left(2^{K} x^{\prime}\right)$. If $\left|x^{\prime}\right|<1002^{K}$ then $\left\|\sum_{k \in J, k \leq K} \phi_{k}\left(x^{\prime}\right)\right\|_{\infty} \leq c 2^{K}$ and

$$
\left\|\theta_{K} * \sum_{k \in J} \phi_{k}\left(x^{\prime}\right)\right\|_{\infty} \leq\left\|\ddot{\theta}_{K} \cdot \sum_{k \in J} \check{\phi}_{k}(\xi)\right\|_{1} \leq c\left\|\check{\theta}_{K}\right\|_{1} \leq c 2^{K} \text {. }
$$


If, instead, $\left|x^{\prime}\right| \geq 1002^{K}$, then $\sum_{k \in J} \phi_{k}\left(x^{\prime}\right)=\sum_{k \in J, k \leq K} \phi_{k}\left(x^{\prime}\right)$, and so for a suitable $\tilde{x}=\tilde{x}\left(x^{\prime \prime}\right)$ we have that

$$
\begin{aligned}
\left|\sum_{\substack{k \in J \\
k \leq K}} \phi_{k}\left(x^{\prime}\right)-\theta_{K} * \sum_{k \in J} \phi_{k}\left(x^{\prime}\right)\right| & =\left|\int \sum_{\substack{k \in J \\
k \leq K}}\left(\phi_{k}\left(x^{\prime}\right)-\phi_{k}\left(x^{\prime}-x^{\prime \prime}\right)\right) \theta_{K}\left(x^{\prime \prime}\right) d x^{\prime \prime}\right| \\
& \leq c \int \sum_{\substack{k \in J \\
k \leq K}}\left|\phi_{k}^{\prime}(\tilde{x})\right| 2^{-K} \theta_{K}\left(x^{\prime \prime}\right) d x^{\prime \prime} \leq c 2^{-K} /\left(x^{\prime}\right)^{2} .
\end{aligned}
$$

Hence the claim is proved. Now if we write $P_{K}\left(x^{\prime}\right)=2^{-K} /\left(x^{\prime}\right)^{2}+2^{-2 K}$ we have that

$$
\begin{aligned}
\left|\sum_{\substack{k \in J \\
k \leq K}} \phi_{k} * f(x)\right| & \leq c\left\{\operatorname{Sup}_{K}\left|\theta_{K} * \sum_{k \in J} \phi_{k} * f(x)\right|+\operatorname{Sup}_{K}\left|P_{K} * f(x)\right|\right\} \\
& \leq c\{M(H f)(x)+M f(x)\} .
\end{aligned}
$$

Therefore the lemma is proved.

The following are called Carleson operator and Carleson maximal operator:

$$
\begin{aligned}
& C f(x)=\int_{-\pi}^{\pi} \exp \left(i N(x) x^{\prime}\right) / x^{\prime} f\left(x-x^{\prime}\right) d x^{\prime}, \\
& \tilde{C} f(x)=\operatorname{Sup}_{\varepsilon>0} \mid \int_{\varepsilon<\left|x^{\prime}\right| \leq \pi} \exp \left(i N(x) x^{\prime}\right) / x^{\prime} f\left(x-x^{\prime}\right) d x^{\prime},
\end{aligned}
$$

where $N(x)$ is any measurable bounded integer valued function. We have

Proposition 1. The operators $C$ and $\tilde{C}$ are bounded from $L_{p}(T)$ to itself, $1<p<\infty$, with norm independent of $N(x)$. Moreover, the following inequality holds: $\tilde{C} f(x) \leq c\{M f(x)+M(C f)(x)\}$.

PROOF. $C f(x)$ is pointwise dominated by the maximal partial sums operator $\operatorname{Sup}_{n}\left|\int_{\left|x^{\prime}\right| \leq \pi} \exp \left(i n x^{\prime}\right) / x^{\prime} f\left(x-x^{\prime}\right) d x^{\prime}\right|$ (also called Carleson operator) whose boundedness has been proved in [4]. As for $\tilde{C} f(x)$ one might go through Carleson and Hunt's proof and see that it shows that $\tilde{C}$ is also bounded, or observe that (see $[6$, p. 218])

$$
\begin{aligned}
& \tilde{C} f(x) \leq \operatorname{Sup}_{\varepsilon>0, n \in Z}\left|\int_{\varepsilon<\left|x^{\prime}\right| \leq \pi} e^{i n x^{\prime}} / x^{\prime} f\left(x-x^{\prime}\right) d x^{\prime}\right| \\
& \quad \leq c\left\{\operatorname{Sup}_{n}\left\{M\left(e^{i n x^{\prime}} f\left(x^{\prime}\right)\right)(x)+M\left(\left|\int_{\left|x^{\prime \prime}\right| \leq \pi} e^{i n x^{\prime \prime}} / x^{\prime \prime} f\left(x^{\prime}-x^{\prime \prime}\right) d x^{\prime \prime}\right|\right)(x)\right\}\right\} \\
& \quad \leq c\{M f(x)+M(C f)(x)\} .
\end{aligned}
$$

This proves the desired inequality and concludes the proof. 
2. The first variant: $\tilde{H}_{1}$. Let $B \subset N \times N$. We consider the operator

$$
H_{1} f(x, y)=\iint_{(k, h) \in B} \phi_{k}\left(x^{\prime}\right) \phi_{h}\left(y^{\prime}\right) f\left(x-x^{\prime}, y-y^{\prime}\right) d x^{\prime} d y^{\prime}
$$

We have the following

THEOREM 1. $H_{1}$ is a bounded operator on $L_{p}\left(T^{2}\right), 1<p<\infty$, with norm independent of $B$.

ProOF. Since $\hat{\phi}_{k}(\xi) \hat{\phi}_{h}(\eta)$ is mainly supported on $\left\{|\xi| \sim 2^{k},|\eta| \sim 2^{h}\right\}$, as in Lemma 1, one can show by the Marcinkiewicz multiplier theorem that the operator

$$
H_{k_{0} h_{0}}^{1} f(x, y)=\iint \sum_{\substack{(k, h) \in B \\ k \leq k_{0}, h \leq h_{0}}} \phi_{k}\left(x^{\prime}\right) \phi_{h}\left(y^{\prime}\right) f\left(x-x^{\prime}, y-y^{\prime}\right) d x^{\prime} d y^{\prime}
$$

is bounded on $L_{p}$ with norm independent of $k_{0}, h_{0}$ and $B$. By a standard argument one can show that there exists $\lim _{k_{0} \rightarrow \infty, h_{0} \rightarrow \infty} H_{k_{0} h_{0}}^{1} f(x, y)=H_{1} f(x, y)$ in $L_{p}$ norm by checking it on a dense subset of functions $f$. It suffices to consider $f\left(x^{\prime}, y^{\prime}\right)=$ $f_{1}\left(x^{\prime}\right) f_{2}\left(y^{\prime}\right), f_{i}$ smooth. Then $H_{1}$ is bounded on $L_{p}$.

Now we have

THEOREM 2. Let $J_{1}$ and $J_{2}$ be subsets of $N$. Let $B \subset J_{1} \times J_{2}$ be a collection of pairs $(k, h)$ such that:

(i) For every $k$ the section $B_{k}=\left\{h \in J_{2}:(k, h) \in B\right\}$ is a truncation of $J_{2}$ possibly depending upon $k$.

(ii) For every $h$ the section $B_{h}=\left\{k \in J_{1}:(k, h) \in B\right\}$ is a truncation of $J_{1}$ possibly depending upon $h$.

Then the operator

$$
\tilde{H}_{1} f(x, y)=\operatorname{Sup}_{k_{0}, h_{0}}\left|\iint \sum_{\substack{(k, h) \in B \\ k \leq k_{0}, h \leq h_{0}}} \phi_{k}\left(x^{\prime}\right) \phi_{h}\left(y^{\prime}\right) f\left(x-x^{\prime}, y-y^{\prime}\right) d x^{\prime} d y^{\prime}\right|
$$

is bounded from $L_{p}\left(T^{2}\right), 1<p<\infty$, to itself with norm independent of $B$. Moreover, if $\tilde{H}$ is defined as in the preceding section the following inequality holds:

$$
\begin{aligned}
\tilde{H}_{1} f(x, y) \leq c\left\{M_{x^{\prime}} M_{y^{\prime}}\right. & f(x, y)+M_{x^{\prime}} \tilde{H}_{y^{\prime}} f(x, y) \\
& \left.+M_{y^{\prime}} \tilde{H}_{x^{\prime}} f(x, y)+M_{x^{\prime}} M_{y^{\prime}}\left(H_{1} f\right)(x, y)\right\} .
\end{aligned}
$$

ProOF. We consider the convolution kernel

$$
G_{k_{0} h_{0}}\left(x^{\prime}, y^{\prime}\right)=\sum_{\substack{(k, h) \in B \\ k \leq k_{0}, h \leq h_{0}}} \phi_{k}\left(x^{\prime}\right) \phi_{h}\left(y^{\prime}\right)-\theta_{k_{0}}\left(x^{\prime}\right) \theta_{h_{0}}\left(y^{\prime}\right) * \sum_{(k, h) \in B} \phi_{k}\left(x^{\prime}\right) \phi_{h}\left(y^{\prime}\right) .
$$

We claim that $\left|G_{k_{0} h_{0}} * f(x, y)\right|$ is dominated by the right-hand side of (4). To prove it we shall subdivide $T^{2}$ into four regions $R_{i}, i=1, \ldots, 4$, and define $G_{k_{0} h_{0}}^{i}\left(x^{\prime}, y^{\prime}\right)=$ $G_{k_{0} h_{0}}\left(x^{\prime}, y^{\prime}\right) \chi_{R_{i}}\left(x^{\prime}, y^{\prime}\right)$. The first region is defined as $R_{1}=\left\{\left|x^{\prime}\right| \leq 1002^{-k_{0}},\left|y^{\prime}\right| \leq\right.$ $\left.1002^{-h_{0}}\right\}$. Now $\left|G_{k_{0} h_{0}}^{1}\left(x^{\prime}, y^{\prime}\right)\right| \leq c 2^{k_{0}} 2^{h_{0}}$ as in the proof of Lemma 1 . Hence 
$\left|G_{k_{0} h_{0}}^{1} * f(x, y)\right| \leq c M_{x^{\prime}} M_{y^{\prime}} f(x, y)$. The second region is defined as $R_{2}=\left\{\left|x^{\prime}\right|<\right.$ $\left.1002^{-k_{0}},\left|y^{\prime}\right|>1002^{-h_{0}}\right\}$. We have that

$$
\begin{aligned}
\left|G_{k_{0} h_{0}}^{2} * f(x, y)\right| \leq & c 2^{k_{0}} \chi_{\left\{\left|x^{\prime}\right| \leq 1002^{\left.-k_{0}\right\}}\right.}\left(x^{\prime}\right) \sum_{k=k_{0}-1 g 200}^{k_{0}}\left|\sum_{\substack{h \in B_{k} \\
h \leq h_{0}}} \phi_{h}\left(y^{\prime}\right) * f(x, y)\right| \\
& +c 2^{k_{0}} 2^{h_{0}} \chi_{\left\{\left|x^{\prime}\right| \leq 1002^{-k_{0}}\right\}}\left(x^{\prime}\right) \chi_{\left\{\left|y^{\prime}\right| \leq 1002^{-h_{0}}\right\}}\left(y^{\prime}\right) \\
& *\left|\sum_{(k, h) \in B} \phi_{k}\left(x^{\prime}\right) \phi_{h}\left(y^{\prime}\right) * f(x, y)\right| \\
\leq & c\left\{M_{x^{\prime}} \tilde{H}_{y^{\prime}} f(x, y)+M_{x^{\prime}} M_{y^{\prime}}\left(H_{1} f\right)(x, y)\right\} .
\end{aligned}
$$

Similarly we define $R_{3}=\left\{\left|x^{\prime}\right|>1002^{-k_{0}},\left|y^{\prime}\right|<1002^{-h_{0}}\right\}$ and we obtain

$$
\left|G_{k_{0} h_{0}}^{3} * f(x, y)\right| \leq c\left\{M_{y^{\prime}} \tilde{H}_{x^{\prime}} f(x, y)+M_{x^{\prime}} M_{y^{\prime}}\left(H_{1} f\right)(x, y)\right\} .
$$

We are left with $R_{4}=\left\{\left|x^{\prime}\right| \geq 1002^{-k_{0}},\left|y^{\prime}\right| \geq 1002^{-h_{0}}\right\}$. Now

$$
\begin{aligned}
& \left|G_{k_{0} h_{0}}^{4}\left(x^{\prime}, y^{\prime}\right)\right|=\left|\theta_{k_{0}} \theta_{h_{0}} * \sum_{\substack{(k, h) \in B \\
k \leq k_{0}, h \leq h_{0}}} \phi_{k}\left(x^{\prime}\right) \phi_{h}\left(y^{\prime}\right)-\sum_{\substack{(k, h) \in B \\
k \leq k_{0}, h \leq h_{0}}} \phi_{k}\left(x^{\prime}\right) \phi_{h}\left(y^{\prime}\right)\right| \\
& =\mid \iint\left\{\sum_{\substack{(k, h) \in B \\
k \leq k_{0}, h \leq h_{0}}} \phi_{k}\left(x^{\prime}-x^{\prime \prime}\right) \phi_{h}\left(y^{\prime}-y^{\prime \prime}\right)-\phi_{k}\left(x^{\prime}\right) \phi_{h}\left(y^{\prime}\right)\right\} \\
& \times \theta_{k_{0}}\left(x^{\prime \prime}\right) \theta_{h_{0}}\left(y^{\prime \prime}\right) d x^{\prime \prime} d y^{\prime \prime} \\
& \left.\leq \mid \iint \sum_{\substack{(k, h) \in B \\
k \leq k_{0}, h \leq h_{0}}} \phi_{k}\left(x^{\prime}-x^{\prime \prime}\right)\left\{\phi_{h}\left(y^{\prime}-y^{\prime \prime}\right)-\phi_{h}\left(y^{\prime}\right)\right\}\right\} \\
& \times \theta_{k_{0}}\left(x^{\prime \prime}\right) \theta_{h_{0}}\left(y^{\prime \prime}\right) d x^{\prime \prime} d y^{\prime \prime} \\
& +\left|\iint \sum_{\substack{(k, h) \in B \\
k \leq k_{0}, h \leq h_{0}}} \phi_{h}\left(y^{\prime}\right)\left\{\phi_{k}\left(x^{\prime}-x^{\prime \prime}\right)-\phi_{k}\left(x^{\prime}\right)\right\} \theta_{k_{0}}\left(x^{\prime \prime}\right) \theta_{h_{0}}\left(y^{\prime \prime}\right) d x^{\prime \prime} d y^{\prime \prime}\right| \\
& =\bar{G}_{k_{0} h_{0}}^{4}\left(x^{\prime}, y^{\prime}\right)+\overline{\bar{G}}_{k_{0} h_{0}}^{4}\left(x^{\prime}, y^{\prime}\right) \text {. }
\end{aligned}
$$


We observe that

$$
\left|\overline{\bar{G}}_{k_{0} h_{0}}^{4}\left(x^{\prime}, y^{\prime}\right)\right|=\left|\int \sum_{k \leq k_{0}}\left\{\phi_{k}\left(x^{\prime}-x^{\prime \prime}\right)-\phi_{k}\left(x^{\prime}\right)\right\} \theta_{k_{0}}\left(x^{\prime \prime}\right) \sum_{\substack{h \in B_{k} \\ h \leq h_{0}}} \phi_{h}\left(y^{\prime}\right) d x^{\prime \prime}\right| .
$$

Hence

$$
\begin{aligned}
&\left|\overline{\bar{G}}_{k_{0} h_{0}}^{4} * f(x, y)\right| \leq \int \sum_{k \leq k_{0}}\left(\int\left|\phi_{k}\left(x^{\prime}-x^{\prime \prime}\right)-\phi_{k}\left(x^{\prime}\right)\right| \theta_{k_{0}}\left(x^{\prime \prime}\right) d x^{\prime \prime}\right) \\
& \cdot \operatorname{Sup}_{h_{0}}\left|\int \sum_{\substack{h \in B_{k} \\
h \leq h_{0}}} \phi_{h}\left(y^{\prime}\right) f\left(x-x^{\prime}, y-y^{\prime}\right) d y^{\prime}\right| d x^{\prime} \\
& \leq c \int P_{k_{0}}\left(x^{\prime}\right)\left|\tilde{H}_{y^{\prime}} f\left(x-x^{\prime}, y\right)\right| d x^{\prime} \leq c M_{x^{\prime}} \tilde{H}_{y^{\prime}} f(x, y) .
\end{aligned}
$$

Now we write

$$
\begin{aligned}
\bar{G}_{k_{0} h_{0}}^{4}\left(x^{\prime}, y^{\prime}\right)=\iint \sum_{\substack{(k, h) \in B \\
k \leq k_{0}, h \leq h_{0}}}\left\{\phi_{h}\left(y^{\prime}-y^{\prime \prime}\right)-\phi_{h}\left(y^{\prime}\right)\right\}\left\{\phi_{k}\left(x^{\prime}-x^{\prime \prime}\right)-\phi_{k}\left(x^{\prime}\right)\right\} \\
\quad \cdot \theta_{k_{0}}\left(x^{\prime \prime}\right) \theta_{h_{0}}\left(y^{\prime \prime}\right) d x^{\prime \prime} d y^{\prime \prime} \\
+\iint \sum_{\substack{(k, h) \in B \\
k \leq k_{0}, h \leq h_{0}}}\left\{\phi_{h}\left(y^{\prime}-y^{\prime \prime}\right)-\phi_{h}\left(y^{\prime}\right)\right\} \phi_{k}\left(x^{\prime}\right) \theta_{k_{0}}\left(x^{\prime \prime}\right) \theta_{h_{0}}\left(y^{\prime \prime}\right) d x^{\prime \prime} d y^{\prime \prime} \\
=\tilde{G}_{k_{0} h_{0}}^{4}\left(x^{\prime}, y^{\prime}\right)+\tilde{G}_{k_{0} h_{0}}^{4}\left(x^{\prime}, y^{\prime}\right) .
\end{aligned}
$$

We start by studying

$$
\begin{aligned}
\left|\tilde{G}_{k_{0} h_{0}}^{4}\left(x^{\prime}, y^{\prime}\right)\right| \leq \iint \sum_{h \leq h_{0}}\left|\phi_{h}\left(y^{\prime}-y^{\prime \prime}\right)-\phi_{h}\left(y^{\prime}\right)\right| \theta_{h_{0}}\left(y^{\prime \prime}\right) \\
\cdot \sum_{k \leq k_{0}}\left|\phi_{k}\left(x^{\prime}-x^{\prime \prime}\right)-\phi_{k}\left(x^{\prime}\right)\right| \theta_{k_{0}}\left(x^{\prime \prime}\right) d x^{\prime \prime} d y^{\prime \prime} .
\end{aligned}
$$

Therefore, $\left|\tilde{G}_{k_{0} h_{0}}^{4} * f(x, y)\right| \leq c P_{h_{0}}\left(y^{\prime}\right) \cdot P_{k_{0}}\left(x^{\prime}\right) * f(x, y) \leq c M_{x^{\prime}} M_{y^{\prime}} f(x, y)$.

We are left to study the action of

$$
\tilde{\tilde{G}}_{k_{0} h_{0}}^{4}\left(x^{\prime}, y^{\prime}\right)=\int \sum_{h \leq h_{0}}\left\{\phi_{h}\left(y^{\prime}-y^{\prime \prime}\right)-\phi_{h}\left(y^{\prime}\right)\right\} \theta_{h_{0}}\left(y^{\prime \prime}\right) \sum_{\substack{k \in B_{h} \\ k \leq k_{0}}} \phi_{k}\left(x^{\prime}\right) d y^{\prime \prime}
$$

Now

$$
\left|\tilde{\tilde{G}}_{k_{0} h_{0}}^{4} * f(x, y)\right| \leq c P_{h_{0}}\left(y^{\prime}\right) \operatorname{Sup}_{k_{0}}\left|\sum_{\substack{k \in B_{h} \\ k \leq k_{0}}} \phi_{k}\left(x^{\prime}\right) * f\right|(x, y) \leq c M_{y^{\prime}} \tilde{H}_{x^{\prime}} f(x, y) .
$$


So the claim has been proved. Now observe that

$$
\begin{aligned}
& \left|\iint \sum_{\substack{(k, h) \in B \\
k \leq k_{0}, h \leq h_{0}}} \phi_{k}\left(x^{\prime}\right) \phi_{h}\left(y^{\prime}\right) f\left(x-x^{\prime}, y-y^{\prime}\right) d x^{\prime} d y^{\prime}\right| \\
& \quad \leq\left|G_{k_{0} h_{0}} * f(x, y)\right|+\left|\theta_{k_{0}}\left(x^{\prime}\right) \theta_{h_{0}}\left(y^{\prime}\right) * \sum_{(k, h) \in B} \phi_{k}\left(x^{\prime}\right) \phi_{h}\left(y^{\prime}\right) * f(x, y)\right| .
\end{aligned}
$$

The last term is dominated by $c M_{x^{\prime}} M_{y^{\prime}}\left(H_{1} f\right)(x, y)$. This ends the proof.

3. The second variant: $\tilde{H}_{2}$. For every $x$ let $B_{x}$ be a fixed subset of $N \times N$ with the following property:

$\left(\right.$ ii $\left.^{\prime}\right)$ for every $h$ there exists an integer $r(x, h)$ such that $B_{x h}=\{k \in N:(k, h) \in$ $\left.B_{x}\right\}=\{k \geq r(x, h)\}$.

We consider the operator

$$
H_{2} f(x, y)=\iint \sum_{(k, h) \in B_{x}} \phi_{h}\left(y^{\prime}\right) \phi_{k}\left(x^{\prime}\right) f\left(x-x^{\prime}, y-y^{\prime}\right) d x^{\prime} d y^{\prime} .
$$

The following theorem holds.

THEOREM 3. In the assumption (ii') there exists a constant $c_{p}$ depending only upon $p$ such that $\left\|H_{2} f\right\|_{p} \leq c_{p}\|f\|_{p}, 1<p<\infty$.

PROOF. We are going to prove that the operator

$$
H_{k_{0} h_{0}}^{2} f(x, y)=\iint \sum_{\substack{(k, h) \in B_{x} \\ k \leq k_{0}, h \leq h_{0}}} \phi_{h}\left(y^{\prime}\right) \phi_{k}\left(x^{\prime}\right) f\left(x-x^{\prime}, y-y^{\prime}\right) d x^{\prime} d y^{\prime}
$$

that for simplicity we also denote by $H_{2} f(x, y)$, is bounded on $L_{p}$ with bound depending only upon $p$. For this purpose it is enough to consider those $f$ which are smooth. The proof is based on (a) and (b) of $\S 1$. Let us denote by $S$ the classical $S$-function acting on functions of one variable, the variable $y$ in our case. By the Littlewood-Paley theorem we know that for a.e. $x$ fixed

$$
\left\|\left(\sum_{J}\left|S_{J} H_{2} f(x, y)\right|^{2}\right)^{1 / 2}\right\|_{L_{p}(d y)} \sim\left\|H_{2} f(x, y)\right\|_{L_{p}(d y)} .
$$

We raise this relation to the $p$ th power and integrate it with respect to $x$, obtaining

$$
\left\|\left(\sum_{J}\left|S_{J} H_{2} f(x, y)\right|^{2}\right)^{1 / 2}\right\|_{L_{p}(d y d x)}^{p} \sim\left\|H_{2} f(x, y)\right\|_{L_{p}(d y d x)}^{p} .
$$

Therefore to prove the boundedness of $H_{2}$ it suffices to prove that

$$
\left\|\left(\left.\sum_{J} S_{J} H_{2} f(x, y)\right|^{2}\right)^{1 / 2}\right\|_{L_{p}(d y d x)} \leq c_{p}\|f\|_{p}
$$


Let $\eta$ denote the dual variable of $y$. In estimating the left-hand side of (5) we consider the dyadic intervals $J$ of each half line separately. Assume from now on that $J$ belongs to $\{\eta \geq 0\}$. For a.e. $x$ fixed we have that

$$
\begin{aligned}
S_{J} H_{2} f(x, y) & =\int_{J} e^{i \eta y} \sum_{h \leq h_{0}} \hat{\phi}_{h}(\eta) \sum_{k \geq r(x, h)} \phi_{k}\left(x^{\prime}\right) * f(x, y)(\eta) d n \\
& =\int_{J} e^{i \eta y} \sum_{h \leq h_{0}}\left\{\int_{2^{\bar{h}}}^{\eta}\left(\hat{\phi}_{h}\right)^{\prime}(t) d t+\hat{\phi}_{h}\left(2^{\bar{h}}\right)\right\} \sum_{k \geq r(x, h)} \phi_{k}\left(x^{\prime}\right) * \hat{f}(x, \eta) d \eta \\
& =S_{J}^{(1)} H_{2} f(x, y)+S_{J}^{(2)} H_{2} f(x, y) .
\end{aligned}
$$

Let $J=\left[2^{\bar{h}}, 2^{\bar{h}+1}\right), h \gtreqless 0$. By exchanging the order of integration we have

(6) $\left|S_{J}^{(2)} H_{2} f(x, y)\right| \leq \sum_{h \leq h_{0}}\left|\hat{\phi}_{h}\left(2^{\bar{h}}\right)\right| \cdot\left|\sum_{k \geq r(x, h)} \phi_{k}\left(x^{\prime}\right) * S_{J} f(x, y)\right| \leq c \tilde{H} S_{J} f(x, y)$.

Next we turn to

$$
S_{J}^{(1)} H_{2} f(x, y)=\int_{J} \sum_{h \leq h_{0}}\left(\hat{\phi}_{h}\right)^{\prime}(t)\left\{\int_{t}^{2^{\bar{h}+1}} e^{i \eta y} \sum_{k \geq r(x, h)} \phi_{k}\left(x^{\prime}\right) * \hat{f}(x, \eta) d \eta\right\} d t .
$$

Now for every $t \in J$ let us denote by $S_{t}$ the multiplier transformation corresponding to the interval $\left[t, 2^{\bar{h}+1}\right)$. Using the relation $S_{t} S_{J}=S_{t}$, Schwarz inequality and (b) we obtain that

$$
\begin{aligned}
\left|S_{J}^{(1)} H_{2} f(x, y)\right| & \leq \int_{J} \sum_{h}\left|\left(\hat{\phi}_{h}\right)^{\prime}(t)\right| \cdot\left|\sum_{k \geq r(x, h)} \phi_{k}\left(x^{\prime}\right) * S_{t} S_{J} f(x, y)\right| d t \\
& \leq c\left(\int_{J} \sum_{h}\left|\left(\hat{\phi}_{h}\right)^{\prime}(t)\right| \cdot\left|\tilde{H} S_{t} S_{J} f(x, y)\right|^{2} d t\right)^{1 / 2} .
\end{aligned}
$$

If we write $d \gamma(t)=\sum_{h}\left|\hat{\phi}_{h}(t)\right| d t$ and we use Theorem $4^{\prime \prime}$ on p. 103 of [7], the Littlewood-Paley theorem, Theorem 1 of [9] and Proposition 2 of [10] we obtain

$$
\begin{aligned}
& \left\|\left(\sum_{J}\left|S_{J}^{(1)} H_{2} f(x, y)\right|^{2}\right)^{1 / 2}\right\|_{L_{p}(d y d x)}^{p} \\
& \leq c\left\|\left(\int_{0}^{+\infty}\left|\tilde{H}_{x^{\prime}} S_{t} S_{J} f(x, y)\right|^{2} d \gamma(t)\right)^{1 / 2}\right\|_{L_{p}(d y d x)}^{p} \\
& \leq c_{p}\left\|\left(\sum_{J}\left|S_{J} f\left(x^{\prime}, y\right)\right|^{2}\right)^{1 / 2}\right\|_{L_{p}(d y d x)}^{p} \leq c_{p}\left\|f_{2}\right\|_{p}^{p} .
\end{aligned}
$$


Now we observe that

$$
\begin{aligned}
& \left\|\left(\sum_{J}\left|S_{J} H_{2} f(x, y)\right|^{2}\right)^{1 / 2}\right\|_{L_{p}(d y d x)}^{p} \\
& \leq\left\|\left(\sum_{J}\left|S_{J}^{(1)} H_{2} f(x, y)\right|^{2}\right)^{1 / 2}+\left(\sum_{J}\left|S_{J}^{(2)} H_{2} f(x, y)\right|^{2}\right)^{1 / 2}\right\|_{L_{p}(d y d x)}^{p} .
\end{aligned}
$$

Now if we use (6) and (7) we obtain (5). So we proved that the operators $H_{k_{0} h_{0}}^{2}$ are uniformly bounded on $L_{p}, 1<p<\infty$. Then to show that there exists

$$
\lim _{k_{0}, h_{0} \rightarrow \infty} H_{k_{0} h_{0}}^{2} f(x, y)=H_{2} f(x, y)
$$

in $L_{p}$ norm it is enough to apply $H_{k_{0} h_{0}}^{2}$ to $f\left(x^{\prime}, y^{\prime}\right)=f_{1}\left(x^{\prime}\right) f_{2}\left(y^{\prime}\right)$ where $f_{i}$ are smooth. Finally one can prove that $\mathrm{H}_{2}$ is bounded on $L_{p}$.

Then we consider the operator

$$
\tilde{H}_{2} f(x, y)=\operatorname{Sup}_{h_{0}}\left|\iint \sum_{\substack{(k, h) \in B_{x} \\ h \leq h_{0}}} \phi_{h}\left(y^{\prime}\right) \phi_{k}\left(x^{\prime}\right) f\left(x-x^{\prime}, y-y^{\prime}\right) d x d y^{\prime}\right| .
$$

We have

THEOREM 4. If (ii') is satisfied then there exists a constant $c_{p}$ depending only upon $p$ such that $\left\|\tilde{H}_{2} f\right\|_{p} \leq c_{p}\|f\|_{p}, 1<p<\infty$. Moreover, the following inequality holds:

$$
\tilde{H}_{2} f(x, y) \leq c\left\{M_{y^{\prime}} \tilde{H}_{x^{\prime}} f(x, y)+M_{\bar{y}}\left(H_{2} f(x, \bar{y})\right)(y)\right\} .
$$

PROOF. We observe that in the formula defining $H_{2} f(x, y)$, once the convolution on the $x^{\prime}$ variable has been performed, the operator acting on the $y^{\prime}$ variable is a constant coefficients singular integral. Hence proceeding as in the proof of Theorem 2 one can show the stated inequality. As a consequence $\tilde{H}_{2}$ is bounded on $L_{p}$.

4. A counterexample. It would be useful for the applications to be able to use the operator

$$
\tilde{H}_{3} f(x, y)=\operatorname{Sup}_{B}\left|\iint \sum_{(k, h) \in B} \phi_{h}\left(y^{\prime}\right) \phi_{k}\left(x^{\prime}\right) f\left(x-x^{\prime}, y-y^{\prime}\right) d x^{\prime} d y^{\prime}\right|
$$

with $B$ an arbitrary subset of $N \times N$. Unfortunately such an operator is unbounded even on $L_{2}$ as it is shown by the following counterexample which has been pointed out to us by C. Fefferman. Consider the operator

$$
K f(x)=\sum_{k \in B_{x}} \phi_{k} * f(x)
$$

We are going to show how to define $B_{x}$ such that $K f(x)=\infty$ at every $x$ for a suitable $f$ belonging to $L_{2}(T)$. Let $f \sim \sum_{n=1}^{\infty}\left\{\exp \left(i \lambda_{n} x\right)\right\} / n$, where the $\lambda_{n}$ 's are sparse, for instance $\lambda_{n}=2^{2^{n}}$. Observe that $\phi_{\lambda_{n}} * f(x)=c_{0}\left\{\exp \left(i \lambda_{n} x\right)\right\} / n+o\left(2^{-n}\right)$, 
where $c_{0}=\hat{\phi}_{\lambda_{n}}\left(\lambda_{n}\right)=\hat{\phi}(1) \neq 0$ as we may assume. Let us subdivide $\{\theta: 0<\theta \leq$ $2 \pi\}$ into four disjoint quadrants $Q_{1}, \ldots, Q_{4}$, where $Q_{1}=\{\theta:-\pi / 4<\theta \leq \pi / 4\}$. For every $x$ fixed there exists at least one of the $Q_{i}$ 's say $Q_{1}$, which contains infinitely many $\lambda_{n} x, n=1,2, \ldots$, and such that $\sum_{\lambda_{n} x \in Q_{1}} 1 / n=\infty$. Define $B_{x}=\{k=$ $\left.\lambda_{n}: \lambda_{n} x \in Q_{1}\right\}$. We have that $|\operatorname{Re}(\exp (i k x))|$ or $|\operatorname{Im}(\exp (i k x))|>c$ for every $k \in B_{x}$. So $\left|\sum_{k \in B_{x}} \phi_{k} * f(x)\right|=\infty$.

5. An application. These results have an application to the study of the operator

$$
T f(x, y)=\iint \exp \left\{i\left(N(x, y) x^{\prime}+N^{2}(x, y) y^{\prime}\right)\right\} / x^{\prime} y^{\prime} f\left(x-x^{\prime}, y-y^{\prime}\right) d x^{\prime} d y^{\prime},
$$

where $N(x, y)$ is a measurable bounded integer valued function. $T f(x, y)$ is the maximal partial sums operator $\operatorname{Sup}_{N}\left|S_{N, N^{2}} f(x, y)\right|$, where

$$
S_{N, N^{2}} f(x, y)=\sum_{\substack{|n| \leq N \\|m| \leq N^{2}}} a_{n m} \cdot \exp \{i(n x+m y)\}
$$

To prove a.e. convergence of $S_{N, N^{2}} f(x, y)$ one looks for an estimate of $T f(x, y)$ which does not depend upon $N(x, y)$. In [5] we proved a uniform estimate in $L_{p}$ of $T f(x, y)$ for $N(x, y)=[\lambda x y], \lambda>10^{10}$, using Theorems 1 and 2 . Moreover, we gave an example of a family of functions $N(x, y)$ which cannot be handled by Theorems 1 and 2 and which leads instead to $\mathrm{H}_{2}$ and $\tilde{H}_{2}$. Let us finally observe that the boundedness of $\tilde{H}_{1}$ and $\tilde{H}_{2}$ is enough for the estimate of pairs of norm 1 while (1) and (2) are needed for the estimate of pairs of norm smaller than 1.

\section{REFERENCES}

1. L. Carleson, On the convergence and growth of partial sums of Fourier series, Acta Math. 116 (1966), 135-157.

2. C. Fefferman, Pointwise convergence of Fourier series, Ann. of Math. (2) 98 (1973), 551-572.

3. R. Fefferman and E. M. Stein, Singular integrals on product spaces, Adv. in Math. 45 (1982), 117-143.

4. R. Hunt, On the convergence of Fourier series, Proc. Conf. on Orthogonal Expansions and their Continuous Analogues, Carbondale Press, Carbondale, Ill., 1968.

5. E. Prestini, Uniform estimates for families of singular integrals and double Fourier series, J. Austral. Math. (to appear).

6. E. M. Stein and G. Weiss, Introduction to Fourier analysis on Euclidean spaces, Princeton Univ. Press, Princeton, N.J., 1971.

7. E. M. Stein, Singular integrals and differentiability properties of functions, Princeton Univ. Press, Princeton, N.J., 1970.

8. P. Sjölin, Convergence almost everywhere of certain singular integrals and multiple Fourier series, Ark. Mat. 9 (1971), 65-90.

9. C. Fefferman and E. M. Stein, Some maximal inequalities, Amer. J. Math. 93 (1971), 107-115.

10. A. Cordoba and C. Fefferman, $A$ weighted norm inequality for singular integrals, Studia Math. 57 (1976), 97-101.

Dipartimento di Matematica, Università Degli Studi, Via Saldini 50, 20133 MILANO, ITALY 